ESAIM: COCV 19 (2013) 555-573

DOI: $10.1051 / \mathrm{cocv} / 2012021$
ESAIM: Control, Optimisation and Calculus of Variations

www.esaim-cocv.org

\title{
LOWER SEMICONTINUITY IN BV OF QUASICONVEX INTEGRALS WITH SUBQUADRATIC GROWTH
}

\author{
PARTh SONEJI ${ }^{1}$
}

\begin{abstract}
A lower semicontinuity result in $B V$ is obtained for quasiconvex integrals with subquadratic growth. The key steps in this proof involve obtaining boundedness properties for an extension operator, and a precise blow-up technique that uses fine properties of Sobolev maps. A similar result is obtained by Kristensen in [Calc. Var. Partial Differ. Equ. 7 (1998) 249-261], where there are weaker asssumptions on convergence but the integral needs to satisfy a stronger growth condition.
\end{abstract}

Mathematics Subject Classification. 49J45.

Received September 30, 2011. Revised March 2, 2012.

Published online March 14, 2013.

\section{INTRODUCTION}

We consider the variational integral

$$
F(u ; \Omega):=\int_{\Omega} f(D u(x)) \mathrm{d} x,
$$

where $\Omega$ is a bounded open subset of $\mathbb{R}^{n}, u: \Omega \rightarrow \mathbb{R}^{N}$ is a vector-valued function, $D u$ denotes the Jacobian matrix of $u$ and $f$ is a nonnegative continuous function defined in the space $\mathbb{R}^{N \times n}$ of all $N \times n$ matrices.

The aim of this paper is to prove a lower semicontinuity result for a quasiconvex integral with an integrand $f$ of subquadratic growth at infinity. Recall that a continuous function $f$ is quasiconvex if for each $\xi \in \mathbb{R}^{N \times n}$ we have

$$
\int_{\mathbb{R}^{n}}[f(\xi+D \phi(x))-f(\xi)] \mathrm{d} x \geq 0
$$

for all test functions $\phi \in C_{0}^{\infty}\left(\mathbb{R}^{n} ; \mathbb{R}^{N}\right)$. We require that $f$ satisfies the following growth condition for $1<r<2$ :

$$
0 \leq f(\xi) \leq L\left(|\xi|^{r}+1\right)
$$

for a fixed finite $L>0$ and all $\xi \in \mathbb{R}^{N \times n}$. Note that (1.2) implies that $F$ is defined and continous on the Sobolev Space $W^{1, r}\left(\Omega ; \mathbb{R}^{N}\right)$.

\footnotetext{
Keywords and phrases. Lower semicontinuity, quasiconvex integrals, functions of bounded variation.

1 Mathematical Institute, University of Oxford, 24-29 St Giles' Oxford, OX1 3LB, UK. soneji@maths.ox.ac.uk;

Parth.Soneji@maths.ox.ac.uk
} 
The following theorem is our main result:

Theorem 1.1. Let $f: \mathbb{R}^{N \times n} \rightarrow \mathbb{R}$ be a continuous quasiconvex function satisfying the growth condition (1.2) for some exponent $1<r<2$. Let $\Omega$ be an open subset of $\mathbb{R}^{n}$.

Let $\left(u_{j}\right)$ be a sequence in $W_{\mathrm{loc}}^{1, r}\left(\Omega ; \mathbb{R}^{N}\right)$ and $u \in W_{\mathrm{loc}}^{1, p}\left(\Omega ; \mathbb{R}^{N}\right)$, where $p \geq 1$ and $p>\frac{r}{2}(n-1)$. Suppose

$$
u_{j} \stackrel{*}{\rightarrow} u \text { in } B V_{\mathrm{loc}}\left(\Omega ; \mathbb{R}^{N}\right)
$$

and

$$
\left(u_{j}\right) \text { uniformly bounded in } L_{\mathrm{loc}}^{q}\left(\Omega ; \mathbb{R}^{n}\right) \text {, }
$$

where

$$
q>\max \left\{1, \frac{r(n-1)}{2-r}\right\}
$$

Then

$$
\liminf _{j \rightarrow \infty} \int_{\Omega} f\left(D u_{j}\right) \mathrm{d} x \geq \int_{\Omega} f(D u) \mathrm{d} x .
$$

Note that when $n \geq 3$, the conditions of this theorem require that the limit map $u$ is more regular than the maps $\left(u_{j}\right)$. When $n=2$, however, we can take $u \in W_{\text {loc }}^{1,1}\left(\Omega ; \mathbb{R}^{N}\right)$, so in this case $u$ can be less regular than the $u_{j}$. In this case, however, there is a result by Kristensen [19] even for $u \in B V$ : see Theorem 2.1. By (1.4) we mean simply that for any compact set $K \subset \Omega$, the sequence $\left(u_{j}\right)$ is uniformly bounded in $L^{q}\left(K ; \mathbb{R}^{N}\right)$, i.e. $\sup _{j}\left\|u_{j}\right\|_{L^{q}(K)} \leq C(K)$, where $C(K)$ is a positive constant possibly depending on $K$. We may remark that this is a natural condition if, for example, we assume that the maps $u_{j}$ and $u$ are constrained to remain on a compact manifold (in which case we would infer the stronger condition that the $\left(u_{j}\right)$ are uniformly bounded in $L^{\infty}\left(\Omega ; \mathbb{R}^{N}\right)$. Indeed, many problems in materials science involve such constrained variational problems - see for instance $[10])$.

The proof of this Theorem 1.1 relies on the following lemma (which is essentially the theorem in the special case where the limit $u$ is affine and where $\Omega$ is the open unit ball $B$ in $\mathbb{R}^{n}$ ) combined with a precise blow-up technique which will be detailed later in this paper.

Lemma 1.2. Let $B$ denote the open unit ball in $\mathbb{R}^{n}$. Suppose $\left(u_{j}\right) \subset W^{1, r}\left(B ; \mathbb{R}^{N}\right), 1<r<2$, and $f: \mathbb{R}^{N \times n} \rightarrow$ $\mathbb{R}$ is as above. Suppose the following conditions hold:

$$
u_{j} \rightarrow 0 \text { strongly in } L^{1}\left(B ; \mathbb{R}^{N}\right)
$$

$$
\sup _{j} \int_{B}\left|D u_{j}\right| \mathrm{d} x<\infty
$$

(iii) There exists a set $F \subset(0,1)$ such that for all $0<\delta<1,|F \cap(\delta, 1)|>0$ and

$$
\sup _{j} \sup _{\varrho \in F}\left\|u_{j}\right\|_{L^{q}\left(\partial B_{\varrho}\right)}<\infty
$$

where

$$
q>\max \left\{1, \frac{r(n-1)}{2-r}\right\}
$$

Then we have the following inequality:

$$
\liminf _{j \rightarrow \infty} \int_{B} f\left(D u_{j}\right) \mathrm{d} x \geq \mathscr{L}^{n}(B) \cdot f(0) .
$$


The proof of this lemma relies on a technique originating in works by Malý et al. (see [11,12,21,24]). A key step in this proof involves obtaining an integral estimate for a trace-preserving extension operator. The result, contained in the following lemma, involves adapting and generalising a result by Carozza et al. [7]. In the statement of this result, as well as subsequently, we denote by $B_{\varrho}$ the open ball in $\mathbb{R}^{n}$ with centre 0 , radius $\varrho$.

Lemma 1.3. Let $1<r<2$. Then for $q$ satisfying

$$
q \geq \max \left\{1, \frac{r(n-1)}{2-r}\right\}
$$

there exists a linear extension operator

$$
\mathbf{E}:\left(W^{1,1} \cap L^{q}\right)\left(\partial B ; \mathbb{R}^{N}\right) \rightarrow W^{1, r}\left(B_{2} \backslash \bar{B} ; \mathbb{R}^{N}\right)
$$

with the following properties:

1. if $g \in C^{1}\left(\partial B ; \mathbb{R}^{N}\right)$ then $\mathbf{E}(g) \in C^{\infty}\left(B_{2} \backslash \bar{B}\right)$ with $\left.\mathbf{E}(g)\right|_{\partial B}=g$;

2. if $\left(z_{j}\right) \subset C^{\infty}\left(\partial B ; \mathbb{R}^{N}\right)$ and $\lim _{j \rightarrow \infty} \int_{\partial B} z_{j} \cdot \phi \mathrm{d} \mathscr{H}^{n-1}=0$ for all $\phi \in C^{\infty}\left(\partial B ; \mathbb{R}^{N}\right)$, then for any multi-index $\alpha, \partial^{\alpha}\left[\mathbf{E} z_{j}\right] \rightarrow 0$ locally uniformly in $B_{2} \backslash \bar{B}$

3. there exist positive constants $c_{1}, c_{2}$, dependent on $n, N, r$, such that:

(a)

$$
\int_{B_{2} \backslash B}|\mathbf{E}(g)|^{r} \leq c_{1}\|g\|_{L^{q}(\partial B)}^{r} ;
$$

(b)

$$
\int_{B_{2} \backslash B}|D \mathbf{E}(g)|^{r} \leq c_{2}\|g\|_{L^{q}(\partial B)}^{\frac{r}{2}} \cdot\|D g\|_{L^{1}(\partial B)}
$$

for all $g \in C^{1}(\partial B)$.

\section{Preliminary REMARKS}

\subsection{Background}

The classical lower semicontinuity result for quasiconvex integrands, essentially attributable to Morrey (see, for example, [9]) says that the functional $F(u ; \Omega)$ defined in (1.1) is (sequentially) weakly lower semicontinuous in $W^{1, p}\left(\Omega ; \mathbb{R}^{N}\right)$ for $f$ satisfying the growth condition

$$
0 \leq f(\xi) \leq L\left(|\xi|^{q}+1\right)
$$

for a fixed finite $L>0$, all $\xi \in \mathbb{R}^{N \times n}$, and where $1 \leq q \leq p<\infty$.

Remaining in the quasiconvex setting, a key refinement of this result is obtained by Malý in [22]: under the growth condition (2.1) (and imposing additional structure conditions), $F(u ; \Omega)$ is proved to be lower semicontinuous in $W^{1, q}\left(\Omega ; \mathbb{R}^{N}\right)$ with respect to weak convergence in $W^{1, p}\left(\Omega ; \mathbb{R}^{N}\right)$ for $p \geq q-1$. Other related works appear in $[6,13,23]$.

For lower semicontinuity theorems in the $B V$ context, we first refer to the monograph of Ambrosio et al. [3]. Firstly, note that it is not entirely clear how to define $F(u ; \Omega)$ when $u$ is a $B V$ function. Following a method that was first used by Lebesgue for the area integral, and then adopted by Serrin $[28,29]$ and, in the modern context, by Marcellini [23], we may consider the functional

$$
\mathscr{F}(u, \Omega):=\inf _{\left(u_{j}\right)}\left\{\begin{array}{l|l}
\liminf _{j \rightarrow \infty} \int_{\Omega} f\left(D u_{j}\right) \mathrm{d} x & \begin{array}{l}
\left(u_{j}\right) \subset C^{1}\left(\Omega, \mathbb{R}^{N}\right) \\
u_{j} \rightarrow u \text { in } L_{\mathrm{loc}}^{1}\left(\Omega, \mathbb{R}^{N}\right)
\end{array}
\end{array}\right\}
$$


$\mathscr{F}$ is known as the Lebesgue-Serrin extension of $F$ and is an important quantity not only when we want to define $F(u ; \Omega)$ for a wider class of functions $u$ but also, for example, when there is a lack of quasiconvexity. In a paper of Ambrosio and Dal Maso [2], it is proved that if $f$ has linear growth at infinity (so $q=1$ in $(2.1)$ ), then for every open set $\Omega \subset \mathbb{R}^{n}$ and every $u \in B V\left(\Omega ; \mathbb{R}^{N}\right)$, we have

$$
\mathscr{F}(u ; \Omega)=\int_{\Omega} f(\nabla u) \mathrm{d} x+\int_{\Omega} f_{\infty}\left(\frac{D^{s} u}{\left|D^{s} u\right|}\right)\left|D^{s} u\right|,
$$

where $\nabla u$ is the density of the absolutely continuous part of the measure $D u$ with respect to Lebesgue measure, $D^{s} u$ is the singular part of $D u, \frac{D^{s} u}{D^{s} u \mid}$ is the Radon-Nikodym derivative of the measure $D^{s} u$ with respect to its variation $\left|D^{s} u\right|$, and $f_{\infty}: \mathbb{R}^{N \times n} \rightarrow[0, \infty)$ is the recession function of $f$ defined by

$$
f_{\infty}(\xi):=\limsup _{t \rightarrow \infty} \frac{f(t \xi)}{t}
$$

In this connection see also [15], where the case of general integrands $f=f(x, u, \nabla u)$ of linear growth is treated, and [26] for a proof that avoids the use of Alberti's rank-one theorem.

For lower semicontinuity theorems in the superlinear growth case, we have the following result by Kristensen [19], which obtains a lower bound for a suitable Lebesgue-Serrin extension. This is similar to Theorem 1.1 and indeed provided the initial motivation for this paper. Here there are weaker assumptions on convergence and on the maps $u_{j}, u$, but the integrand $f$ needs to satisfy a stronger growth condition.

Theorem 2.1. [19] Let $f: \mathbb{R}^{N \times n} \rightarrow \mathbb{R}$ be a continuous quasiconvex function satisfying the growth condition

$$
0 \leq f(\xi) \leq L\left(|\xi|^{q}+1\right)
$$

for a fixed finite $L>0$, a fixed exponent $q \in\left[1, \frac{n}{n-1}\right)$, and all $\xi \in \mathbb{R}^{N \times n}$. Let $\Omega$ be an open, bounded subset of $\mathbb{R}^{n}$.

Suppose $\left(u_{j}\right) \subset W_{\text {loc }}^{1, q}\left(\Omega ; \mathbb{R}^{N}\right)$ converges to $u \in B V_{\text {loc }}\left(\Omega ; \mathbb{R}^{N}\right)$ in $L_{\text {loc }}^{1}\left(\Omega ; \mathbb{R}^{N}\right)$ and

$$
\sup _{j} \int_{\omega}\left|D u_{j}\right|<+\infty \text { for all } \omega \subset \subset \Omega \text {. }
$$

Then

$$
\liminf _{j \rightarrow \infty} \int_{\Omega} f\left(D u_{j}\right) \mathrm{d} x \geq \int_{\Omega} f(\nabla u) \mathrm{d} x
$$

where $\nabla u$ denotes the approximate gradient of $u$.

For related work in the compensated compactness set-up, see also [16]. Also of importance is the paper of Carozza et al. [7] where a similar condition to (1.4) in Theorem 1.1 is used to obtain a lower semicontinuity result. Namely, for an integrand $f$ satisfying $(2.1)$, where $q=p+1$ and $p>1$, we have lower semicontinuity with respect to sequences of functions $\left(u_{j}\right)$ in $W_{\text {loc }}^{1, p+1}\left(\Omega ; \mathbb{R}^{N}\right)$ that converge weakly to $u$ in $W_{\text {loc }}^{1, p}\left(\Omega ; \mathbb{R}^{N}\right)$, provided also that $u_{j}-u$ converges to 0 strongly in $L_{\text {loc }}^{\infty}\left(\Omega ; \mathbb{R}^{N}\right)$ and $u \in C^{1}\left(\Omega ; \mathbb{R}^{N}\right)$. Finally we mention that the theory discussed here is not vacuous. Indeed it has been shown by Šverák [30] that there exist quasiconvex functions on $\mathbb{R}^{2 \times 2}$ that have subquadratic growth and are not polyconvex (and hence not convex). In this connection we also mention $[25,31]$. 


\subsection{General remarks on the result}

The results of this paper can actually be stated with the following more general subquadratic growth condition:

Let $\Phi:[0, \infty) \rightarrow[0, \infty)$ be a convex, doubling, non-decreasing function such that $\Phi(0)=0$ and, for some $\sigma_{\Phi}>0$ :

$$
t \mapsto \frac{\Phi(t)}{t^{2-\sigma_{\Phi}}} \text { is nonincreasing on }(0, \infty) \text { and } \int_{1}^{\infty} \frac{\Phi(t)^{\frac{1}{2}}}{t^{2}} \mathrm{~d} t<\infty .
$$

(Recall that $\Phi$ is doubling means that, for a fixed constant $c, \Phi(2 t) \leq c \Phi(t)$ for all $t \geq 0$ ).

Assume that $f$ satisfies

$$
0 \leq f(\xi) \leq L(\Phi(|\xi|)+1)
$$

for a fixed finite $L>0$ and all $\xi \in \mathbb{R}^{N \times n}$.

This growth condition implies that $F$ is defined and continous on the generalised Orlicz-Sobolev space $W^{1, \Phi}\left(\Omega ; \mathbb{R}^{N}\right)$. For further information on the subject of such spaces, we refer to the book of Iwaniec and Martin [18]. We have focused in particular on the case where $\Phi$ is of the form $\Phi(t)=t^{r}$, which just puts us in the more familiar setting of the Sobolev Space $W^{1, r}\left(\Omega ; \mathbb{R}^{N}\right)$, noting that for such $\Phi,(2.2)$ is satisfied whenever $1<r<2$.

Our result for subquadratic quasiconvex integrands hinges on a result by Greco et al. in [17], concerning integral estimates of the Hardy-Littlewood Maximal function. In this connection the condition (2.2) is sharp and the proof provided cannot be weakened to include integrands of quadratic growth at infinity. Indeed, we do not know if the main theorem is true for $f$ satisfying (1.2) for $r=2$, even when $\Omega=B, u=0, u_{j} \stackrel{*}{\rightarrow} 0$ in $B V\left(B ; \mathbb{R}^{N}\right)$ and $u_{j} \rightarrow 0$ in $L^{\infty}\left(B ; \mathbb{R}^{N}\right)$. However, what is clear is that the proof of such a result, if it is true, needs to proceed by a different means. We may deduce from the following counterexample, established by Malý in [20], that the result is certainly not true when $f$ has at least cubic growth in some directions (and $n, N \geq 3$ ).

Counterexample $2.2([20])$. Let $Q$ be the cube $(0,1)^{n}$, and $1<r<n-1$. There is a sequence of orientationpreserving $C^{1}$-diffeomorphisms $\left(u_{j}\right)$ on $Q$ such that $u_{j}$ converge weakly to the identity in $W^{1, r}\left(Q, \mathbb{R}^{n}\right)$ and

$$
\lim _{j \rightarrow \infty} \int_{Q} \operatorname{det} D u_{j} \mathrm{~d} x=0 .
$$

A suitable counterexample for our purposes immediately follows by taking $n=3$ (or, if we want a result in higher dimensions we can simply consider a suitable $3 \times 3$ minor of the Jacobian), $\Omega=Q$, and $f(\xi)=|\operatorname{det} \xi|$. $f$ is polyconvex, hence quasiconvex, and satisfies the growth condition

$$
0 \leq f(\xi) \leq L\left(|\xi|^{3}+1\right) .
$$

Moreover the $u_{j}$, being diffeomorphisms of $Q$ onto $Q$, are clearly uniformly bounded in $L^{q}\left(Q, \mathbb{R}^{n}\right)$ for any $1 \leq q \leq \infty$, and weak convergence in $W^{1, r}$ for $1<r<2$ obviously implies weak* convergence in $B V$. And if $u$ is the identity map on $Q$, then $\int_{Q} \operatorname{det} D u \mathrm{~d} x=1$. So all the conditions of Theorem 1.1 except the growth condition are satisfied, but lower semicontinuity does not obtain.

It is interesting to note that we can also set the main result of this paper in the context of $W^{1,1}$-quasiconvexity. The condition of $W^{1, p}$-quasiconvexity, which generalises in a natural way the quasiconvexity condition of Morrey, is defined and studied in the well-known paper of Ball and Murat [4]. Namely, a continuous integrand $f$ is said to be $W^{1, p}$-quasiconvex (where $1 \leq p \leq \infty$ ) if it is bounded below and satisfies

$$
\int_{\mathbb{R}^{n}}[f(\xi+D \phi(x))-f(\xi)] \mathrm{d} x \geq 0
$$

for all $\xi \in \mathbb{R}^{N \times n}$ and $\phi \in W_{0}^{1, p}\left(\mathbb{R}^{n} ; \mathbb{R}^{N}\right)$. If $p=\infty$ then this condition is just the usual definition of quasiconvexity. In the Proof of Lemma 1.2, we use the well known result that the conditions on $f$ in Theorem 1.1, in 
particular its continuity, quasiconvexity and growth condition (1.2), imply that it is $W^{1, r}$-quasiconvex. Crucially, this is why we need the maps $u_{j}$ in Theorem 1.1 to be in $W_{\text {loc }}^{1, r}\left(\Omega ; \mathbb{R}^{N}\right)$. However, if we assume the stronger condition that $f$ is $W^{1,1}$-quasiconvex, we only require the $\left(u_{j}\right)$ to be in $W_{\text {loc }}^{1,1}\left(\Omega ; \mathbb{R}^{N}\right)$. That is, we have the following result, which has virtually the same proof as Theorem 1.1.

Theorem 2.3. Let $f: \mathbb{R}^{N \times n} \rightarrow \mathbb{R}$ be a continuous $W^{1,1}$-quasiconvex function satisfying the growth condition (1.2) for some exponent $1<r<2$. Let $\Omega$ be an open subset of $\mathbb{R}^{n}$.

Let $\left(u_{j}\right)$ be a sequence in $W_{\mathrm{loc}}^{1,1}\left(\Omega ; \mathbb{R}^{N}\right)$ and $u \in W_{\mathrm{loc}}^{1, p}\left(\Omega ; \mathbb{R}^{N}\right)$, where $p \geq 1$ and $p>\frac{r}{2}(n-1)$. Suppose

$$
u_{j} \stackrel{*}{\rightarrow} u \text { in } B V_{\mathrm{loc}}\left(\Omega ; \mathbb{R}^{N}\right)
$$

and

$$
\left(u_{j}\right) \text { uniformly bounded in } L_{\mathrm{loc}}^{q}\left(\Omega ; \mathbb{R}^{n}\right) \text {, }
$$

where

$$
q>\max \left\{1, \frac{r(n-1)}{2-r}\right\}
$$

Then

$$
\liminf _{j \rightarrow \infty} \int_{\Omega} f\left(D u_{j}\right) \mathrm{d} x \geq \int_{\Omega} f(D u) \mathrm{d} x
$$

It is also worth briefly discussing more generally the regularity assumptions of the maps $u_{j}, u$ in the main result. The increased regularity requirement on $u$, that it is in $W_{\text {loc }}^{1, p}\left(\Omega ; \mathbb{R}^{N}\right)$ where $p \geq 1$ and $p>\frac{r}{2}(n-1)$, is required to make use of the fact that the $\left(u_{j}\right)$ are uniformly bounded in $L_{\text {loc }}^{q}\left(\Omega ; \mathbb{R}^{N}\right)$ for $q$ satisfying (1.5) when using the "blow-up argument" to obtain the proof of Theorem 1.1 from Lemma 1.2.

Another issue is that if we just assume that $f$ is quasiconvex in the sense of Morrey, then we could consider whether lower semicontinuity still obtains if the maps $\left(u_{j}\right)$ are less regular than $W_{\mathrm{loc}}^{1, r}\left(\Omega ; \mathbb{R}^{N}\right)$. Even though it is still an open question whether lower semicontinuity obtains when $f$ has quadratic growth, the following counterexample provided in [4] demonstrates (if we take $n=2$ ) that in this case we would certainly require at least that the $\left(u_{j}\right)$ are in $W_{\text {loc }}^{1,2}\left(\Omega ; \mathbb{R}^{N}\right)$.

Counterexample 2.4 ([4]). Let $n>1$ and $\Omega$ be an open bounded subset of $\mathbb{R}^{n}$. Let $1 \leq r<n$. Then there exist $\left(u_{j}\right) \subset W^{1, r}\left(\Omega ; \mathbb{R}^{n}\right)$ such that $u_{j}$ converge weakly in $W^{1, r}\left(\Omega ; \mathbb{R}^{n}\right)$ to the identity map, as well as strongly in $L^{\infty}\left(\Omega ; \mathbb{R}^{n}\right)$ (hence the $\left(u_{j}\right)$ are uniformly bounded in $L^{\infty}\left(\Omega ; \mathbb{R}^{n}\right)$ ), but for all $j, \operatorname{det} D u_{j}(x)=0$ for almost all $x \in \Omega$.

Let us now consider properties of a suitable Lebesgue-Serrin extension, and introduce the functional (for $1<r<2$ and $q$ satisfying $(1.5))$

$$
\mathscr{F}(u, \Omega):=\inf _{\left(u_{j}\right)}\left\{\begin{array}{l|l}
\liminf _{j \rightarrow \infty} \int_{\Omega} f\left(D u_{j}\right) \mathrm{d} x & \begin{array}{l}
\left(u_{j}\right) \subset W_{\text {loc }}^{1, r}\left(\Omega, \mathbb{R}^{N}\right) \\
\left(u_{j}\right) \text { uniformly bounded in } L_{\mathrm{loc}}^{q}\left(\Omega, \mathbb{R}^{N}\right) \\
u_{j} \stackrel{*}{*} u \text { weakly* in } B V_{\text {loc }}\left(\Omega, \mathbb{R}^{N}\right)
\end{array}
\end{array}\right\} .
$$

Note that Theorem 1.1 implies the following result:

Corollary 2.5. Let $f: \mathbb{R}^{N \times n} \rightarrow \mathbb{R}$, satisfy the conditions in Theorem 1.1. Then

- If $n \geq 3, p \geq 1$ and $p>\frac{r}{2}(n-1)$, and $q$ satisfies $(1.5), \mathscr{F}(u ; \Omega)=F(u ; \Omega)$ for all $u \in\left(W_{\mathrm{loc}}^{1, p} \cap L_{\mathrm{loc}}^{q}\right)\left(\Omega ; \mathbb{R}^{N}\right)$;

- if $n=2$, then this equality holds for all $u \in\left(W_{\mathrm{loc}}^{1, r} \cap L_{\mathrm{loc}}^{q}\right)\left(\Omega ; \mathbb{R}^{N}\right)$. 
Proof. For any $n$, Theorem 1.1 tells us that if $u \in W_{\text {loc }}^{1, p}\left(\Omega ; \mathbb{R}^{N}\right)$ for $p$ satisfying the stated conditions, and $\left(u_{j}\right)$ is a sequence satisfying the conditions given in the definition of $\mathscr{F}(u ; \Omega)$, then

$$
\liminf _{j \rightarrow \infty} \int_{\Omega} f\left(D u_{j}\right) \mathrm{d} x \geq F(u ; \Omega) .
$$

Taking the infimum of all such $\left(u_{j}\right)$, we get

$$
\mathscr{F}(u ; \Omega) \geq F(u ; \Omega) \text { when } u \in W_{\mathrm{loc}}^{1, p}\left(\Omega ; \mathbb{R}^{N}\right) .
$$

Now note that if $u \in\left(W_{\text {loc }}^{1, r} \cap L_{\text {loc }}^{q}\right)\left(\Omega ; \mathbb{R}^{N}\right)$, then by simply taking $u_{j}=u$ for all $j$, we get a sequence satisfying the conditions in $\mathscr{F}(u ; \Omega)$, so certainly

$$
F(u ; \Omega) \geq \mathscr{F}(u ; \Omega) \text { when } u \in\left(W_{\mathrm{loc}}^{1, r} \cap L_{\mathrm{loc}}^{q}\right)\left(\Omega ; \mathbb{R}^{N}\right) .
$$

We conclude by noting that since $1<r<2$, for $n \geq 3$ we have

$$
W_{\mathrm{loc}}^{1, p}\left(\Omega, \mathbb{R}^{N}\right) \subset W_{\mathrm{loc}}^{1, r}\left(\Omega, \mathbb{R}^{N}\right),
$$

and for $n=2$, since in this case we can take $p=1$,

$$
W_{\text {loc }}^{1, r}\left(\Omega, \mathbb{R}^{N}\right) \subset W_{\text {loc }}^{1, p}\left(\Omega, \mathbb{R}^{N}\right) .
$$

If we wish to describe $\mathscr{F}$ for an even wider class of functions $u$, things can be more difficult. Certainly, if $u \notin L_{\mathrm{loc}}^{q}\left(\Omega ; \mathbb{R}^{N}\right)$ then there can be no sequence $\left(u_{j}\right)$ uniformly bounded in $L_{\mathrm{loc}}^{q}\left(\Omega ; \mathbb{R}^{N}\right)$ satisfying $u_{j} \stackrel{*}{\rightarrow} u$ in $B V_{\text {loc }}\left(\Omega ; \mathbb{R}^{N}\right)$ (or even just strongly in $L_{\text {loc }}^{1}$ ), since this would imply that $u$ is itself in $L_{\text {loc }}^{q}\left(\Omega ; \mathbb{R}^{N}\right)$. Hence we have

$$
\mathscr{F}(u, \Omega)=\inf \emptyset=+\infty \text {. }
$$

Results by Bouchitté et al. [5], Fonseca and Malý [11,12] indicate that, even for $n \geq 3$, a measure representation for $\mathscr{F}$ should exist for $u \in\left(W_{\text {loc }}^{1, r} \cap L_{\text {loc }}^{q}\right)\left(\Omega ; \mathbb{R}^{N}\right)$, but we have been unable to prove this yet. A counterexample due to Acerbi and Dal Maso [1] shows that if $r=n=N=2$ and $u \in\left(B V_{\text {loc }} \cap L_{\text {loc }}^{\infty}\right)\left(\Omega ; \mathbb{R}^{N}\right)$, then a measure representation does not exist at all. Although their conditions are slightly different from ours, it is not difficult to see from their paper that their counterexample also applies to our case. In fact, they present an example where the set function $\omega \mapsto \mathscr{F}(u ; \omega)$ is not even subadditive (for an alternative proof, see [8]).

\section{Proof of the MAIN RESUlt}

Proof of Lemma 1.2. By approximation we may assume $\left(u_{j}\right) \subset C^{1}\left(\bar{B} ; \mathbb{R}^{N}\right)$. If the left hand side of (1.11) is infinite then there is nothing to prove, so suppose it is finite. Moreover, by extracting a subsequence if necessary, we can assume

$$
l_{0}:=\liminf _{j \rightarrow \infty} \int_{B} f\left(D u_{j}\right) \mathrm{d} x=\lim _{j \rightarrow \infty} \int_{B} f\left(D u_{j}\right) \mathrm{d} x .
$$

With reference to (1.9), write $M=\sup _{j} \sup _{\varrho \in F}\left\|u_{j}\right\|_{L^{q}\left(\partial B_{\varrho}\right)}$. From (1.7), by the Fubini-Tonelli theorem and the Rellich-Kondrachoff compactness theorem we have

$$
\lim _{j \rightarrow \infty} \int_{0}^{1} \int_{\partial B_{\varrho}}\left|u_{j}\right| \mathrm{d} \mathscr{H}^{n-1} \mathrm{~d} \varrho=\lim _{j \rightarrow \infty} \int_{B}\left|u_{j}\right| \mathrm{d} x=0 .
$$

This implies there exists a subsequence $\left\{u_{j}\right\}_{j \in T}$ such that

$$
\lim _{j \rightarrow \infty, j \in T} \int_{\partial B_{\varrho}}\left|u_{j}\right| \mathrm{d} \mathscr{H}^{n-1}=0
$$


for almost all $\varrho \in(0,1)$. By Fatou's Lemma and (1.8) we have

$$
\int_{0}^{1} \liminf _{j \rightarrow \infty, j \in T} \int_{\partial B_{\varrho}}\left|D u_{j}\right| \mathrm{d} \mathscr{H}^{n-1} \mathrm{~d} \varrho \leq \liminf _{j \rightarrow \infty, j \in T} \int_{B}\left|D u_{j}\right| \mathrm{d} x<\infty .
$$

Thus, for almost all $\varrho \in(0,1)$

$$
\liminf _{j \rightarrow \infty, j \in T} \int_{\partial B_{\varrho}}\left|D u_{j}\right| \mathrm{d} \mathscr{H}^{n-1}<\infty .
$$

Now fix $0<\delta<1$. By (3.1), (3.2) and (1.9) we can choose $\varrho \in(\delta, 1)$ such that all the following hold: 1.

2 .

$$
\lim _{j \rightarrow \infty, j \in T} \int_{\partial B_{\varrho}}\left|u_{j}\right| \mathrm{d} \mathscr{H}^{n-1}=0
$$

$$
\liminf _{j \rightarrow \infty, j \in T} \int_{\partial B_{\varrho}}\left|D u_{j}\right| \mathrm{d} \mathscr{H}^{n-1}<\infty
$$

3.

$$
\sup _{j \in T}\left\|u_{j}\right\|_{L^{q}\left(\partial B_{\varrho}\right)} \leq M .
$$

Now take a further subsequence $\left\{u_{j}\right\}_{j \in S}$, where $S \subseteq T$, so that

$$
\lim _{j \rightarrow \infty, j \in S} \int_{\partial B_{\varrho}}\left|D u_{j}\right| \mathrm{d} \mathscr{H}^{n-1}=\liminf _{j \rightarrow \infty, j \in T} \int_{\partial B_{\varrho}}\left|D u_{j}\right| \mathrm{d} \mathscr{H}^{n-1} .
$$

Relabel the sequence $\left(u_{j}\right)$ so that $S=\mathbb{N}$. Now define the sequence $\left(g_{j}\right) \subset W^{1,1}\left(\partial B ; \mathbb{R}^{N}\right)$ as:

$$
g_{j}(x):=\left.u_{j}\right|_{\partial B_{\varrho}}(\varrho x) \text { for } x \in \partial B .
$$

Take a cut-off function $\eta \in C^{1}(B ; \mathbb{R})$ such that $\mathbf{1}_{B_{\varrho}} \leq \eta \leq \mathbf{1}_{B},|D \eta| \leq \frac{2}{1-\varrho}$, and define $\left(v_{j}\right) \subset W_{0}^{1, r}\left(B ; \mathbb{R}^{N}\right)$ as:

$$
v_{j}(x):=\left\{\begin{array}{cc}
\eta(x) \cdot\left(\mathbf{E}\left(g_{j}\right)\right)\left(\frac{x}{\varrho}\right) & \text { if }|x| \geq \varrho, \\
u_{j}(x) & \text { if }|x|<\varrho,
\end{array}\right.
$$

where $\mathbf{E}$ is the extension operator from Lemma 1.3.

Since the function $t \mapsto t^{r}$ is convex, $(s+t)^{r} \leq 2^{r-1}\left(s^{r}+t^{r}\right)$ for all $s, t \geq 0$. Hence from Lemma 1.3 we have

$$
\begin{aligned}
\int_{B \backslash B_{\varrho}}\left|D v_{j}\right|^{r} & \leq \int_{B \backslash B_{\varrho}}\left(\left|D \eta \cdot \mathbf{E} g_{j}(\cdot / \varrho)\right|+\left|\eta \cdot D\left[\mathbf{E} g_{j}(\cdot / \varrho)\right]\right|\right)^{r} \\
& \leq 2^{r-1} \int_{B \backslash B_{\varrho}}|D \eta|^{r} \cdot\left|\mathbf{E} g_{j}(\cdot / \varrho)\right|^{r}+2^{r-1} \int_{B \backslash B_{\varrho}}|\eta|^{r} \cdot\left|D\left[\mathbf{E} g_{j}(\cdot / \varrho)\right]\right|^{r} \\
& \leq C \int_{B \backslash B_{\varrho}}\left|\mathbf{E} g_{j}(\cdot / \varrho)\right|^{r}+C \int_{B \backslash B_{\varrho}}\left|D\left[\mathbf{E} g_{j}(\cdot / \varrho)\right]\right|^{r}
\end{aligned}
$$

for some constant C. We estimate the two terms in (3.3) using Lemma 1.3 (3) as follows:

$$
\begin{aligned}
\int_{B \backslash B_{\varrho}}\left|D\left[\mathbf{E} g_{j}(\cdot / \varrho)\right]\right|^{r} & \leq c_{2}\left\|g_{j}\right\|_{L^{q}(\partial B)}^{\frac{r}{2}} \cdot\left\|D g_{j}\right\|_{L^{1}(\partial B)} \\
\text { by }(1.9) & \leq c_{2} M^{\frac{r}{2}} \cdot\left\|D g_{j}\right\|_{L^{1}(\partial B)} \\
& =C \int_{\partial B_{\varrho}}\left|D u_{j}\right| \mathrm{d} \mathscr{H}^{n-1}
\end{aligned}
$$


for another constant C. Now note that we may obtain the same inequality (albeit for a different constant $C$ ) using Lemma 1.3 for any other $r^{\prime}$ such that $r<r^{\prime}<2$, and also (with reference to (1.10)) satisfying $q>\frac{r^{\prime}(n-1)}{2-r^{\prime}}$. Hence by (3.4) and Lemma 1.3, since

$$
\sup _{j} \int_{\partial B_{\varrho}}\left|D u_{j}\right| \mathrm{d} \mathscr{H}^{n-1}<\infty
$$

we can use the De la Vallée Poussin criterion to deduce that the sequence $\left|D\left[\mathbf{E} g_{j}\right]\right|^{r}$ is equi-integrable on $B \backslash B_{\varrho}$. By Lemma 1.3, since

$$
\sup _{j} \int_{\partial B_{\varrho}}\left|u_{j}\right| \mathrm{d} \mathscr{H}^{n-1} \rightarrow 0 \text { as } j \rightarrow \infty,
$$

$D\left[\mathbf{E} g_{j}\right] \rightarrow 0$ locally uniformly on $B \backslash B_{\varrho}$, and hence so does $\left|D\left[\mathbf{E} g_{j}\right]\right|^{r}$. Thus, by Vitali's Convergence Theorem,

$$
\int_{B \backslash B_{\varrho}}\left|D\left[\mathbf{E} g_{j}(\cdot / \varrho)\right]\right|^{r} \rightarrow 0 \quad \text { as } j \rightarrow \infty .
$$

Similarly

$$
\begin{aligned}
\int_{B \backslash B_{\varrho}}\left|\left[\mathbf{E} g_{j}(\cdot / \varrho)\right]\right|^{r} & \leq c_{1}\left\|g_{j}\right\|_{L^{q}(\partial B)}^{r} \\
\operatorname{by}(1.9) & \leq c_{1} M^{r}
\end{aligned}
$$

so $\left|\mathbf{E} g_{j}\right|^{r}$ is equi-integrable on $B \backslash B_{\varrho}$, and using Lemma 1.3 and Vitali,

$$
\int_{B \backslash B_{\varrho}}\left|\mathbf{E} g_{j}(\cdot / \varrho)\right|^{r} \rightarrow 0 \quad \text { as } j \rightarrow \infty .
$$

Combining these estimates in (3.3), we have

$$
\limsup _{j \rightarrow \infty} \int_{B \backslash B_{\varrho}}\left|D v_{j}\right|^{r} \mathrm{~d} x=0 .
$$

Now we use the quasiconvexity and nonnegativity of $f$ to obtain

$$
\begin{aligned}
\int_{B} f\left(D u_{j}\right) & \geq \int_{B_{\varrho}} f\left(D u_{j}\right)=\int_{B} f\left(D v_{j}\right)-\int_{B \backslash B_{\varrho}} f\left(D v_{j}\right) \geq \mathscr{L}^{n}(B) f(0)-\int_{B \backslash B_{\varrho}} f\left(D v_{j}\right) \\
& \geq \mathscr{L}^{n}(B) f(0)-L \int_{B \backslash B_{\varrho}}\left(1+\left|D v_{j}\right|^{r}\right) .
\end{aligned}
$$

Let $j \rightarrow \infty$ to get, using (3.5),

$$
l_{0} \geq \mathscr{L}^{n}(B) f(0)-L \mathscr{L}^{n}\left(B \backslash B_{\varrho}\right) .
$$

Recall $\varrho \in(\delta, 1)$ for fixed $0<\delta<1$. Hence we conclude by taking $\delta$ arbitrarily close to 1 .

In order to obtain the proof of Theorem 1.1 from Lemma 1.2, we use a technique originating in work by Foneseca and Müller, which was further developed by Fonseca and Marcellini (see [13,14]). However, this "blowup argument" still does not apply completely for our purposes. In order to use the fact the sequence $\left(u_{j}\right)$ in Theorem 1.1 is uniformly bounded in $L_{\text {loc }}^{q}\left(\Omega ; \mathbb{R}^{N}\right)$ for $q$ satisfying (1.5), we need to be more careful in our choice of blow-up functions. This involves applying the following lemma. 
Lemma 3.1. Let $u \in W_{\text {loc }}^{1, p}\left(\Omega ; \mathbb{R}^{N}\right)$, where $1 \leq p<n-1$. Then for almost all $x_{0} \in \Omega$ the following holds: there exists a set $E \subset(0,1)$ such that 0 is a point of right density one of $E$, and the difference quotient

$$
\int_{\partial B_{\varrho}}\left(\frac{\left|u\left(x_{0}+z\right)-u\left(x_{0}\right)-\left[D u\left(x_{0}\right)\right] z\right|}{|z|}\right)^{\frac{(n-1) p}{n-1-p}} \mathrm{~d} \mathscr{H}^{n-1}(z)
$$

tends to 0 as $\varrho \rightarrow 0$ through the set $E$. Moreover, the set $E$ has the following property: there exists a sequence $t_{k} \searrow 0$ and corresponding sets $E_{t_{k}} \subset\left[\frac{1}{2}, 1\right]$ such that

$$
E=\bigcup_{i=1}^{\infty} t_{i} E_{t_{i}}
$$

and, for any $\epsilon>0$, we can choose $t_{k}, E_{t_{k}}$ such that

$$
\left|\bigcap_{i=1}^{\infty} E_{t_{i}}\right|>\frac{1}{2}-\epsilon
$$

Proof of Lemma 3.1. For $x_{0}, y \in \Omega, t>0$, define

$$
v(t, y):=\frac{u\left(x_{0}+t y\right)-u\left(x_{0}\right)-\left[D u\left(x_{0}\right)\right](t y)}{t} .
$$

It is clear that, provided $B\left(x_{0}, t\right) \subset \Omega, v(t, y) \in W^{1, p}\left(B ; \mathbb{R}^{N}\right)$. Moreover, it is well known that $\int_{B}|v(t, y)|^{p} \mathrm{~d} y \rightarrow 0$ as $t \rightarrow 0$ for almost all $x_{0} \in \Omega$ (see, for example, [32]). In addition, by considering Lebesgue points of $D u$, we have

$$
\int_{B}|D v(t, y)|^{p} \mathrm{~d} y=\int_{B}\left|D u\left(x_{0}+t y\right)-D u\left(x_{0}\right)\right|^{p} \mathrm{~d} y \longrightarrow 0 \text { as } t \rightarrow 0
$$

for almost all $x_{0} \in \Omega$. Fix such an $x_{0}$ and, for $0<t<\operatorname{dist}\left(x_{0}, \partial \Omega\right)$, define

$$
\gamma(t):=\int_{\partial B}|v(t, y)|^{\frac{(n-1) p}{n-1-p}} \mathrm{~d} \mathscr{H}^{n-1}(y),
$$

and

$$
\alpha(t):=\int_{B}\left(|v(t, y)|^{p}+|D v(t, y)|^{p}\right) \mathrm{d} y .
$$

Note that by our choice of $x_{0}$ we have $\alpha(t) \rightarrow 0$ as $t \rightarrow 0$. Since $v(t, y) \in W^{1, p}(B)$ for $t$ sufficiently small, we have $v(t, y) \in W^{1, p}\left(\partial B_{\varrho} ; \mathbb{R}^{N}\right)$ for almost all $\varrho \in(0,1)$. It then follows by the Rellich-Kondrachoff embedding theorem that in fact $v(t, y) \in L^{\frac{(n-1) p}{n-1-p}}\left(\partial B_{\varrho} ; \mathbb{R}^{N}\right)$ for almost all $\varrho \in(0,1)$.

Now let

$$
\phi_{t}(\varrho):=\int_{\partial B_{\varrho}}\left(|v(t, y)|^{p}+|D v(t, y)|^{p}\right) \mathrm{d} \mathscr{H}^{n-1}(y)
$$

and let

$$
E_{t}:=\left[\frac{1}{2}, 1\right] \cap\left\{\varrho: \phi_{t}(\varrho)<\alpha(t)^{\frac{1}{2}}\right\} .
$$

Note

$$
\alpha(t)=\int_{0}^{1} \phi_{t}(\varrho) \mathrm{d} \varrho \geq \int_{\frac{1}{2}}^{1} \phi_{t}(\varrho) \mathrm{d} \varrho \geq \int_{\left[\frac{1}{2}, 1\right] \backslash E_{t}} \phi_{t}(\varrho) \mathrm{d} \varrho \geq\left|\left[\frac{1}{2}, 1\right] \backslash E_{t}\right| \cdot \alpha(t)^{\frac{1}{2}},
$$

so

$$
\left|\left[\frac{1}{2}, 1\right] \backslash E_{t}\right| \leq \alpha(t)^{\frac{1}{2}} .
$$


Next consider $\varrho \in E_{t}$. By the Sobolev Inequality we have for $\beta=\frac{(n-1) p}{n-1-p}$ and some constant $M=M(p, n)$ :

$$
\left(\int_{\partial B_{\varrho}}|v(y, t)|^{\beta} \mathrm{d} \mathscr{H}^{n-1}(y)\right)^{\frac{1}{\beta}} \leq M\left(\int_{\partial B_{\varrho}}|v(y, t)|^{p}+|D v(t, y)|^{p} \mathrm{~d} \mathscr{H}^{n-1}(y)\right)^{\frac{1}{p}}=M \phi_{t}(\varrho)^{\frac{1}{p}} \leq M \alpha(t)^{\frac{1}{2 p}} .
$$

Hence, again for $\varrho \in E_{t}$, we have

$$
\begin{aligned}
\gamma(\varrho t) & =\int_{\partial B}\left|\frac{u\left(x_{0}+\varrho t y\right)-u\left(x_{0}\right)-\left[D u\left(x_{0}\right)\right](\varrho t y)}{\varrho t}\right|^{\beta} \mathrm{d} \mathscr{H}^{n-1}(y) \\
& =\varrho^{1-n-\beta} \int_{\partial B_{\varrho}}|v(t, y)|^{\beta} \mathrm{d} \mathscr{H}^{n-1}(y) \\
& \leq M 2^{n+\beta+1} \cdot \alpha(t)^{\frac{n-1}{2(n-1-p)}} .
\end{aligned}
$$

Now we may take any decreasing sequence $\left(t_{i}\right) \subset\left(0, \operatorname{dist}\left(x_{0}, \partial \Omega\right)\right)$ such that $t_{i} \searrow 0$ and let $E_{t_{i}}$ be defined as in (3.8). Note that we could also require $t_{i+1}<t_{i} / 2$, so that the $E_{t_{i}}$ are disjoint. Now define $E$ as stated in (3.7). Thus 0 is a limit point of $E$, and $\gamma(\varrho) \rightarrow 0$ as $r \rightarrow 0, \varrho \in E$, so the main statement of the lemma is proved.

It remains to show that we can choose $\left(t_{i}\right)$ such that $\left|\bigcap_{i=1}^{\infty} E_{t_{i}}\right|$ is arbitrarily close to $\frac{1}{2}$. Write $E_{t}^{c}$ for $\left[\frac{1}{2}, 1\right] \backslash E_{t}$. Since $E_{t}^{c} \searrow 0$ as $t \searrow 0$, for a given $\epsilon>0$ we may choose $t_{i} \searrow 0$ such that $E_{t_{i}}^{c}<2^{-i} \epsilon$ for all $i$. Hence

$$
\left|\bigcup_{i=1}^{\infty} E_{t_{i}}^{c}\right| \leq \sum_{i=1}^{\infty}\left|E_{t_{i}}^{c}\right|<\epsilon
$$

So

$$
\left|\bigcap_{i=1}^{\infty} E_{t_{i}}\right|>\frac{1}{2}-\epsilon
$$

This completes the proof of the lemma.

Remark 3.2. If $u \in W_{\mathrm{loc}}^{1, p}\left(\Omega ; \mathbb{R}^{N}\right)$ for $p \geq n-1$, then obviously $u \in W_{\mathrm{loc}}^{1, p^{\prime}}\left(\Omega ; \mathbb{R}^{N}\right)$ for any $1 \leq p^{\prime} \leq p$, so we can still apply the above lemma for $1 \leq p^{\prime}<n-1$ to prove Theorem 1.1. In fact, if $p>n-1$, then we have a stronger result: namely that $u$ has a regular approximate total differential at almost all $x_{0} \in \Omega$. This means that the difference quotient

$$
\frac{\left|u\left(x_{0}+z\right)-u\left(x_{0}\right)-\left[D u\left(x_{0}\right)\right] z\right|}{|z|}
$$

tends to 0 uniformly for $z \in \partial B_{\varrho}$ as $\varrho \rightarrow 0$ through a set $E$ for which 0 is a point of right density one. A scheme of a proof of this can be found in [32] (Chap. 3, Exercises), from which the Proof of Lemma 3.1 has been adapted.

Proof of Theorem 1.1. We may assume that the left hand side of (1.6) is finite, as otherwise there is nothing to prove. Taking a subsequence if necessary, we can also assume that

$$
\int_{\Omega} f\left(D u_{j}\right) \rightarrow \liminf _{j \rightarrow \infty} \int_{\Omega} f\left(D u_{j}\right)
$$

For our purposes, for (1.3) we require only the following fact which characterizes weak* convergence in $B V$. That is, (1.3) is equivalent to:

$$
\begin{aligned}
u_{j} \rightarrow u & \text { in } L_{\mathrm{loc}}^{1}\left(\Omega ; \mathbb{R}^{N}\right) \\
D u_{j} \stackrel{*}{\rightarrow} D u & \text { in } \mathscr{M}\left(\Omega ; \mathbb{R}^{N \times n}\right),
\end{aligned}
$$


where $\mathscr{M}\left(\Omega ; \mathbb{R}^{N \times n}\right)$ is the space of $N \times n$ matrix-valued Borel measures on $\Omega$. By (3.10) and the Uniform Boundedness Principle, $\sup _{j} \int_{\Omega}\left|D u_{j}\right| \mathrm{d} \mathscr{L}^{n}<\infty$. Since $\mathscr{L}^{n}\left\lfloor f\left(D u_{j}\right)\right.$ and $\mathscr{L}^{n}\left\lfloor\left|D u_{j}\right|\right.$ are bounded in $\mathscr{M}\left(\bar{\Omega} ; \mathbb{R}^{N \times n}\right)$, we that have for some subsequence (for convenience not relabelled) there exist measures $\mu$ and $\nu$ in $\bar{\Omega}$ such that

$$
\text { and } \left.\begin{array}{r}
f\left(D u_{j}\right) \stackrel{*}{\rightarrow} \mu \\
\left|D u_{j}\right| \stackrel{*}{\rightarrow} \nu
\end{array}\right\} \quad \text { in } \mathscr{M}\left(\bar{\Omega} ; \mathbb{R}^{N \times n}\right) .
$$

Notice that, because $f \geq 0$, the proof of the theorem follows if we can prove that

$$
\frac{\mathrm{d} \mu}{\mathrm{d} \mathscr{L}^{n}}(x) \geq f(D u(x))
$$

holds for almost all $x \in \Omega$.

Let $\Omega_{0}$ denote the set of points $x \in \Omega$ such that:

1.

2 .

$$
\frac{\mathrm{d} \mu}{\mathrm{d} \mathscr{L}^{n}}(x)=\lim _{\varrho \rightarrow 0^{+}} \frac{\mu\left(\overline{\mathcal{L}^{n}(x, \varrho)}\right)}{B(x, \varrho))} \quad \text { exists and is finite; }
$$

$$
\frac{\mathrm{d} \nu}{\mathrm{d} \mathscr{L}^{n}}(x)=\lim _{\varrho \rightarrow 0^{+}} \frac{\nu\left(\overline{\mathscr{L}^{n}(x, \varrho)}\right)}{(B(x, \varrho))} \quad \text { exists and is finite; }
$$

3.

$$
\lim _{\varrho \rightarrow 0^{+}} \frac{1}{\varrho} f_{B(x, \varrho)}|u(y)-u(x)-[D u(x)](x-y)| \mathrm{d} y=0 ;
$$

4. Lemma 3.1 holds for $u$ at $x$.

By standard results (see e.g. [27,32]) and Lemma 3.1, $\Omega_{0}$ has full measure in $\Omega$. Fix $x_{0} \in \Omega_{0}$. Let $\left(r_{k}\right) \subset$ $\left(0, \operatorname{dist}\left(x_{0}, \Omega\right)\right)$ be a sequence such that $r_{k} \searrow 0$ and define

$$
v_{j, k}(y):=\frac{u_{j}\left(x_{0}+r_{k} y\right)-u\left(x_{0}\right)-\left[D u\left(x_{0}\right)\right]\left(r_{k} y\right)}{r_{k}}, \quad y \in B .
$$

Our aim is to pick a suitable sequence $\left(r_{k}\right)$ so we may use $v_{j, k}$ to define a sequence $\left(z_{k}\right) \subset W^{1, r}\left(B ; \mathbb{R}^{N}\right)$, say, that will enable us to apply Lemma 1.2 to obtain (3.11). In fact, we do not actually apply Lemma 1.2 for the same $q$ as in Theorem 1.1, but for an arbitrarily smaller $q^{\prime}<q$ that nevertheless satisfies (1.10).

However, also note that in order to apply Lemmas 1.2 and 3.1 , from (1.10) and (3.6) we need $u \in W_{\text {loc }}^{1, p}\left(\Omega ; \mathbb{R}^{N}\right)$ for $1 \leq p<n-1$ satisfying

$$
\frac{p(n-1)}{n-1-p}>\max \left\{1, \frac{r(n-1)}{2-r}\right\} \text {. }
$$

It is straightforward to verify that this holds if and only if $1 \leq p<n-1$ also satisfies $p>\frac{r}{2}(n-1)$.

Recall from Lemma 3.1 that, for any $\epsilon>0$, we can choose a sequence $t_{k} \searrow 0, t_{k}<\operatorname{dist}\left(x_{0}, \partial \Omega\right)$, such that $\left|\bigcap E_{t_{k}}\right|>\frac{1}{2}-\epsilon$, with $E_{t_{k}}$ defined as in (3.8). By (1.4) and (3.9), using De la Vallée Poussin and Vitali, we have $u_{j} \rightarrow u$ in $L_{\text {loc }}^{q^{\prime}}(\Omega)$ for any $1 \leq q^{\prime}<q$. Hence, for any fixed $k$ :

$$
\lim _{j \rightarrow \infty} \frac{1}{t_{k}^{q^{\prime}}} \int_{B}\left|u_{j}\left(x_{0}+t_{k} y\right)-u\left(x_{0}+t_{k} y\right)\right|^{q^{\prime}} \mathrm{d} y=0
$$

So, by Fubini-Tonelli,

$$
\lim _{j \rightarrow \infty} \frac{1}{t_{k}^{q^{\prime}}} \int_{0}^{1} \int_{\partial B_{\varrho}}\left|u_{j}\left(x_{0}+t_{k} y\right)-u\left(x_{0}+t_{k} y\right)\right|^{q^{\prime}} \mathrm{d} \mathscr{H}^{n-1}(y) \mathrm{d} \varrho=0 .
$$


Hence, for every $k$, there exists a subsequence $\left(u_{j}\right)_{j \in S_{k}}, S_{k} \subseteq \mathbb{N}$, such that

$$
\lim _{j \rightarrow \infty, j \in S_{k}} \frac{1}{t_{k}^{q^{\prime}}} \int_{\partial B_{\varrho}}\left|u_{j}\left(x_{0}+t_{k} y\right)-u\left(x_{0}+t_{k} y\right)\right|^{q^{\prime}} \mathrm{d} \mathscr{H}^{n-1}(y)=0
$$

for almost all $\varrho \in(0,1)$. Now note that, by Egorov's Theorem, for a given $\epsilon>0$, there exists a set $G_{k} \subset(0,1)$ such that $\left|(0,1) \backslash G_{k}\right|<\epsilon 2^{-k}$ and (3.13) holds uniformly for $\varrho \in G_{k}$. By discarding smaller elements of $S_{k}$ if necessary, this implies that

$$
\sup _{j \in S_{k}} \sup _{\varrho \in G_{k}} \frac{1}{t_{k}^{q^{\prime}}} \int_{\partial B_{\varrho}}\left|u_{j}\left(x_{0}+t_{k} y\right)-u\left(x_{0}+t_{k} y\right)\right|^{q^{\prime}} \mathrm{d} \mathscr{H}^{n-1}(y)<1 .
$$

We can obtain such $G_{k}$ and $S_{k}$ for all $k \in \mathbb{N}$. Now note that we have, similarly to the remark to Lemma 3.1, $\left|\bigcap G_{k}\right|>1-\epsilon$, so $\left|\left(\frac{1}{2}, 1\right) \cap \bigcap G_{k}\right|>\frac{1}{2}-\epsilon$. Therefore

$$
\left|\bigcap_{k=1}^{\infty}\left(G_{k} \cap E_{t_{k}}\right)\right|>\frac{1}{2}-2 \epsilon>0,
$$

provided $\epsilon$ is small enough. This means that $\bigcap\left(G_{k} \cap E_{t_{k}}\right)$ contains a point of left density one $\theta$, say (so $\theta \in\left(\frac{1}{2}, 1\right)$ ). If we let

$$
F=\theta^{-1} \bigcap_{k=1}^{\infty}\left(G_{k} \cap E_{t_{k}}\right)
$$

then 1 is a point of left density one of $F$. Hence, for all $0<\delta<1,|(\delta, 1) \cap F|>0$.

Now let $r_{k}=\theta t_{k}$. So $\left(r_{k}\right) \subset\left(0, \operatorname{dist}\left(x_{0}, \Omega\right)\right), r_{k} \searrow 0$. Note also that the set

$$
\left\{\varrho \in\left(0, \operatorname{dist}\left(x_{0}, \Omega\right)\right):(\mu+\nu)\left(\partial B\left(x_{0}, \varrho\right)\right)>0\right\}
$$

is at most countable, so has measure 0 . Since there are uncountably many points of left density one, like $\theta$, above, we may assume in addition that $(\mu+\nu)\left(\partial B\left(x_{0}, r_{k}\right)\right)=0$ for all $k$ for our choice of $r_{k}$. Define $v_{j, k}$ in $(3.12)$ using this choice of $r_{k}$. Observe that we may also write $v_{j, k}$ as follows:

$$
v_{j, k}(y)=\frac{1}{r_{k}}\left(u\left(x_{0}+r_{k} y\right)-u\left(x_{0}\right)-\left[D u\left(x_{0}\right)\right]\left(r_{k} y\right)\right)+\frac{1}{r_{k}}\left(u_{j}\left(x_{0}+r_{k} y\right)-u\left(x_{0}+r_{k} y\right)\right)=I+I I, \text { say. }
$$

We now consider $I$ and $I I$ separately.

Estimating I. If $\varrho \in F$, then $\theta \varrho \in \bigcap E_{t_{k}}$, so $\varrho \theta t_{k} \in t_{k} E_{t_{k}}$ for all $k$. i.e. $\varrho r_{k} \in t_{k} E_{t_{k}}$ for all $k$. So, with reference to (3.7) in Lemma 3.1, we have that $\varrho r_{k} \in E$ : so if $y \in \partial B$, then $\varrho r_{k} y \in A$. So by Lemma 3.1 we have

$$
\begin{gathered}
\sup _{\varrho \in F} \int_{\partial B}\left(\frac{\left|u\left(x_{0}+\varrho r_{k} y\right)-u\left(x_{0}\right)-\left[D u\left(x_{0}\right)\right]\left(\varrho r_{k} y\right)\right|}{\left|\varrho r_{k}\right|}\right)^{\frac{(n-1) p}{n-1-p}} \mathrm{~d} \mathscr{H}^{n-1}(y) \\
\leq C \cdot \alpha\left(t_{k}\right)^{\frac{n-1}{2(n-1-p)}} \longrightarrow 0 \text { as } k \rightarrow 0 .
\end{gathered}
$$

This implies that

$$
\sup _{k} \sup _{\varrho \in F}\left\|\frac{1}{r_{k}}\left(u\left(x_{0}+r_{k} \cdot\right)-u\left(x_{0}\right)-\left[D u\left(x_{0}\right)\right]\left(r_{k} \cdot\right)\right)\right\|_{L_{q^{\prime}\left(\partial B_{\varrho}\right)}}<\infty
$$

for any $1 \leq q^{\prime} \leq \frac{(n-1) p}{n-1-p}$. Hence, as noted above, if $p$ satisfies the conditions in Theorem 1.1, then we can choose an appropriate $q^{\prime}$ satisfying (1.10). 
Estimating II. Take a subsequence $\left(u_{j_{k}}\right)$ of $\left(u_{j}\right)$ such that $j_{k} \in S_{k}$ for all $k$. Hence we have for all $\varrho \in F$, $\theta \varrho \in \bigcap G_{k}$ (so indeed $\varrho \theta \in G_{k}$ for every $k$ ). So, for any $1 \leq q^{\prime}<q$,

$$
\begin{aligned}
& \frac{1}{r_{k}^{q^{\prime}}} \int_{\partial B_{\varrho}}\left|u_{j_{k}}\left(x_{0}+r_{k} y\right)-u\left(x_{0}+r_{k} y\right)\right|^{q^{\prime}} \mathrm{d} \mathscr{H}^{n-1}(y) \\
& \quad=\frac{1}{\left(\theta t_{k}\right)^{q^{\prime}}} \int_{\partial B_{\varrho}}\left|u_{j_{k}}\left(x_{0}+\theta t_{k} y\right)-u\left(x_{0}+\theta t_{k} y\right)\right|^{q^{\prime}} \mathrm{d} \mathscr{H}^{n-1}(y) \\
& \quad=\frac{1}{\left(\theta t_{k}\right)^{q^{\prime}}} \int_{\partial B_{\varrho} \theta}\left|u_{j_{k}}\left(x_{0}+t_{k} y\right)-u\left(x_{0}+t_{k} y\right)\right|^{q^{\prime}} \theta^{1-n} \mathrm{~d} \mathscr{H}^{n-1}(y) \\
& \quad<\theta^{1-n-q^{\prime}}
\end{aligned}
$$

for every $k \in \mathbb{N}, \varrho \in F$. Hence

$$
\sup _{k} \sup _{\varrho \in F}\left\|\frac{1}{r_{k}}\left(u_{j_{k}}\left(x_{0}+r_{k} \cdot\right)-u\left(x_{0}+r_{k} \cdot\right)\right)\right\|_{L^{q^{\prime}}\left(\partial B_{\varrho}\right)}<\infty .
$$

Therefore, combining these two estimates, we have shown that for this subsequence, $\left(v_{j_{k}, k}\right)_{k \in \mathbb{N}}$ satisfies $(1.9)$ of Lemma 1.2 (for $q^{\prime}$ in place of $q$ ).

Now note

$$
\begin{aligned}
f_{B}\left|D v_{j, k}(y)+D u\left(x_{0}\right)\right| \mathrm{d} y & =f_{B}\left|D u_{j}\left(x_{0}+r_{k} y\right)\right| \mathrm{d} y \\
& =\frac{1}{\left|B\left(x_{0}, r_{k}\right)\right|} \int_{B\left(x_{0}, r_{k}\right)}\left|D u_{j}(y)\right| \mathrm{d} y \longrightarrow \frac{\mathrm{d} \nu}{\mathrm{d} \mathscr{L}^{n}}\left(x_{0}\right) \text { as } j, k \rightarrow \infty,
\end{aligned}
$$

and similarly we can take a subsequence $\left(u_{j_{k}}\right)$ such that (by property (2) for $\Omega_{0}$ above), (1.8) of Lemma 1.2 is satisfied.

In the same way,

$$
f_{B} f\left(D v_{j, k}(y)\right) \mathrm{d} y \longrightarrow \frac{\mathrm{d} \mu}{\mathrm{d} \mathscr{L}^{n}}\left(x_{0}\right) \quad \text { as } j, k \rightarrow \infty,
$$

and we can take a subsequence $\left(u_{j_{k}}\right)$ so that this convergence happens as $k \rightarrow \infty$.

Thus, taking multiple subsequences, we can indeed create a sequence $\left(z_{k}\right)=\left(v_{j_{k}, k}\right) \subset W^{1, r}\left(B ; \mathbb{R}^{N}\right)$ satisfying (1.7)-(1.9). Hence, by Lemma 1.2,

$$
\liminf _{k \rightarrow \infty} f\left(D z_{k}(y)+D u\left(x_{0}\right)\right) \mathrm{d} y \geq f\left(D u\left(x_{0}\right)\right)
$$

i.e.

$$
\frac{\mathrm{d} \mu}{\mathrm{d} \mathscr{L}^{n}}\left(x_{0}\right) \geq f\left(D u\left(x_{0}\right)\right)
$$

\section{Proof of integral estimates For the EXtension operator}

In this section we provide a Proof of Lemma 1.3, which provides us with an extension operator that enables us to get higher integrability in the Proof of Lemma 1.2. By a standard localisation argument (see, for example, [24]), it suffices to prove Lemma 4.1 below. We denote points in $\mathbb{R}^{n}$ by $(x, t)$, where $x \in \mathbb{R}^{n-1}$ and $t \in \mathbb{R}$, and let

$$
\mathbb{R}_{+}^{n}:=\left\{(x, t) \in \mathbb{R}^{n}: t>0\right\} .
$$


Lemma 4.1. Let $1<r<2$. Then for $q$ satisfying

$$
q \geq \max \left\{1, \frac{r(n-1)}{2-r}\right\}
$$

there exists a linear extension operator

$$
\mathbf{E}:\left(W_{\text {loc }}^{1,1} \cap L_{\text {loc }}^{q}\right)\left(\mathbb{R}^{n-1} ; \mathbb{R}^{N}\right) \rightarrow W_{\text {loc }}^{1, r}\left(\mathbb{R}_{+}^{n} ; \mathbb{R}^{N}\right)
$$

with the following properties:

1. if $h \in C^{1}\left(\mathbb{R}^{n-1} ; \mathbb{R}^{N}\right)$ then $(\mathbf{E} h)(x, 0):=\lim _{t \rightarrow 0^{+}}(\mathbf{E} h)(x, t)=h(x)$ for all $x \in \mathbb{R}^{n-1}$;

2. if $\left(z_{j}\right) \subset C^{\infty}\left(\mathbb{R}^{n-1} ; \mathbb{R}^{N}\right)$ and $z_{j} \rightarrow 0$ in the sense of distributions, then for any multi-index $\alpha, \partial^{\alpha}\left[\mathbf{E} z_{j}\right] \rightarrow 0$ locally uniformly in $\mathbb{R}_{+}^{n}$;

3. For all $R>0$ there exist positive constants $c_{1}$, $c_{2}$, dependent on $n, N, r, R$, such that for all $h \in\left(W^{1,1} \cap\right.$ $\left.L^{q}\right)\left(\mathbb{R}^{n-1} ; \mathbb{R}^{N}\right)$ with support contained in $\left\{x \in \mathbb{R}^{n-1}:|x| \leq R\right\}$ we have

(a)

$$
\int_{\mathbb{R}_{+}^{n}}|\mathbf{E} h|^{r} \mathrm{~d} \mathscr{L}^{n} \leq c_{1}\|h\|_{L^{q}\left(\mathbb{R}^{n-1}\right)}^{r} ;
$$

$$
\int_{\mathbb{R}_{+}^{n}}|D[\mathbf{E} h]|^{r} \mathscr{L}^{n} \leq c_{2}\|g\|_{L^{q}\left(\mathbb{R}^{n-1}\right)}^{\frac{r}{2}} \cdot \int_{\mathbb{R}^{n-1}}|D h| \mathrm{d} \mathscr{H}^{n-1} .
$$

Since the function $t \mapsto t^{r}$ is convex, it suffices to prove Lemma 4.1 in the case where $N=1$. For convenience, let $m=n-1$, and let $\mathcal{B}^{m}$ denote the open unit ball in $\mathbb{R}^{m}$. To define $\mathbf{E}$, we take a standard convolution kernel $K$ in $\mathbb{R}^{m}$ supported in $\mathcal{B}^{m}$ (i.e. $K$ satisfies $K \in C_{c}^{\infty}\left(\mathbb{R}^{m}\right), K \geq 0, \int K=1, \operatorname{supp}(K) \subset \subset \mathcal{B}^{m}$ ) and a function $\eta \in C_{c}^{\infty}(\mathbb{R})$ with $\eta(0)=1, \eta(1)=0$. Then we let

$$
(\mathbf{E} h)(x, t):=\eta(t)\left(K_{t} * h\right)(x), \quad(x, t) \in \mathbb{R}_{+}^{n} .
$$

It is easily seen that $\mathbf{E}$ maps $\left(W_{\text {loc }}^{1,1} \cap L_{\text {loc }}^{q}\right)\left(\mathbb{R}^{m}\right)$ into $C^{\infty}\left(\mathbb{R}_{+}^{n}\right)$ and that it satisfies properties (1) and (2). In order to show that it maps the given domain into $W_{\text {loc }}^{1, r}\left(\mathbb{R}_{+}^{n}\right)$, it suffices to prove $3(\mathrm{~b})$. We shall prove it for the $x$ derivative $D_{x}(\mathbf{E} h(x, t))$ only, since proving it for the $t$ derivative is entirely similar, concluding for $D(\mathbf{E} h)$ using the convexity of $r \mapsto t^{r}$. Throughout the proofs of 3(a), 3(b), we will use $c$ to denote a constant, not always the same from line to line, that depends at most on $n, r, \eta, K, R, q$.

Proof of $3(a)$. Note that

so

$$
(\mathbf{E} h)(x, t)=\eta(t) \int_{\mathcal{B}^{m}} t^{-m} K\left(\frac{y}{t}\right) h(x-y) \mathrm{d} \mathscr{H}^{m}(y)
$$

$$
|\mathbf{E} h|^{r} \leq\left(c\|\eta\|_{\infty}\|K\|_{\infty} \int_{\mathcal{B}^{m}} t^{-m}|h(x-y)| \mathrm{d} y\right)^{r} .
$$

Now we use Jensen's Inequality to obtain, for any $q \geq 1$,

$$
\begin{aligned}
\int_{t \mathcal{B}^{m}} t^{-m}|h(x-y)| \mathrm{d} y & =\left|t \mathcal{B}^{m}\right| t^{-m} f_{t \mathcal{B}^{m}}|h(x-y)| \mathrm{d} y \\
& =\left|\mathcal{B}^{m}\right|\left(\left(f_{t \mathcal{B}^{m}}|h(x-y)| \mathrm{d} y\right)^{q}\right)^{\frac{1}{q}} \\
& \leq\left|\mathcal{B}^{m}\right|\left(f_{t \mathcal{B}^{m}}|h(x-y)|^{q} \mathrm{~d} y\right)^{\frac{1}{q}} \\
& =t^{-\frac{m}{q}}\left(\int_{t \mathcal{B}^{m}}|h(x-y)|^{q} \mathrm{~d} y\right)^{\frac{1}{q}} \\
& \leq t^{-\frac{m}{q}}\|h\|_{L^{q}\left(\mathbb{R}^{m}\right) .}
\end{aligned}
$$


Hence we have

$$
|\mathbf{E} h|^{r} \leq c\|h\|_{L^{q}\left(\mathbb{R}^{m}\right)}^{r} t^{-\frac{m r}{q}} .
$$

Now note that $\int_{0}^{1} t^{-\frac{m r}{q}} \mathrm{~d} t$ is finite if and only if $\frac{m r}{q}>-1$, i.e. $q>r m$. Since $1<r<2$, this is certainly satisfied if $q \geq \frac{m r}{2-r}$.

Moreover, if $h$ is compactly supported in $\left\{x \in \mathbb{R}^{m}:|x| \leq R\right\}$, then $\mathbf{E} h$ is supported in $\left\{(x, t) \in \mathbb{R}_{+}^{n}:|x| \leq\right.$ $R+1, t \in(0,1)\}$. Hence, integrating $|\mathbf{E} h|^{r}$ first with respect to $t$, then $x$, we get, for $q>r m$,

$$
\int_{\mathbb{R}_{+}^{n}}|\mathbf{E} h|^{r} \mathrm{~d} \mathscr{L}^{n}=\int_{\{|x| \leq R+1\}} \int_{0}^{1}|\mathbf{E} h|^{r} \mathrm{~d} t \mathrm{~d} x \leq c(R+1)^{m}\left|\mathcal{B}^{m}\right|\|h\|_{L^{q}\left(\mathbb{R}^{m}\right)}^{r} \cdot \int_{0}^{1} t^{-\frac{r m}{q}} \mathrm{~d} t \leq c_{1}\|h\|_{L^{q}\left(\mathbb{R}^{m}\right)}^{r} .
$$

The key component of the proof of 3(b) of Lemma 4.1 depends on the following lemma due to Greco et al. (see [17]). Before stating this lemma, we shall establish some definitions. For $g \in L_{\text {loc }}^{1}\left(\mathbb{R}^{m}\right)$ recall that the Hardy-Littlewood maximal function is defined as:

$$
(M g)(x):=\sup _{\varrho>0} f_{\varrho \mathcal{B}^{m}}|g(x-y)| \mathrm{d} y, \quad x \in \mathbb{R}^{m} .
$$

Let $\Phi:[0, \infty) \rightarrow[0, \infty)$ be a non-decreasing, right-continuous function and define the function $\Psi:[0, \infty) \rightarrow[0, \infty)$ as

$$
\Psi(t):=\Phi(t)+t \int_{0}^{t} \frac{\Phi(s)}{s^{2}} \mathrm{~d} s, \quad t \geq 0 .
$$

Lemma $4.2([17])$. Let $\Phi, \Psi:[0, \infty) \rightarrow[0, \infty)$ be defined as above. If $g \in L^{1}\left(\mathbb{R}^{m}\right)$, then

$$
\int_{\mathbb{R}^{m}} \Phi(M g) \mathrm{d} x \leq 2 \cdot 5^{m} \int_{\mathbb{R}^{m}} \Psi(2|g|) \mathrm{d} x .
$$

Proof of $3(b)$. First observe that, by integration by parts and since $K$ vanishes on $\partial \mathcal{B}^{m}$, we may write the derivative $D_{x}(\mathbf{E} h)$ in both the following ways:

$$
D_{x}(\mathbf{E} h)(x, t)=\eta(t) \int_{\mathcal{B}^{m}} D h(x-t y) K(y) \mathrm{d} y=\eta(t) \int_{\mathcal{B}^{m}} t^{-1} h(x-t y) D K(y) \mathrm{d} y .
$$

Now fix $x \in \mathbb{R}^{m}$. Integrating $D_{x}(\mathbf{E} h)$ first with respect to $t$ over $(0,1)$, we get

$$
\begin{aligned}
\int_{0}^{1}\left|D_{x}(\mathbf{E} h)(x, t)\right|^{r} \mathrm{~d} t & =\int_{0}^{\delta}\left(\left|\eta(t) \int_{\mathcal{B}^{m}} D h(x-t y) K(y) \mathrm{d} y\right|\right)^{r} \mathrm{~d} t \int_{\delta}^{1}\left(\left|\eta(t) \int_{\mathcal{B}^{m}} t^{-1} h(x-t y) D K(y) \mathrm{d} y\right|\right)^{r} \mathrm{~d} t \\
& =I+I I, \quad \text { say. }
\end{aligned}
$$

Estimating I. We obtain the following bound on $I$ :

$$
\begin{aligned}
I & =\int_{0}^{\delta}\left(\left|\eta(t) \int_{\mathcal{B}^{m}} D h(x-t y) K(y) \mathrm{d} y\right|\right)^{r} \mathrm{~d} t \\
& \leq \int_{0}^{\delta}\|\eta\|_{\infty}^{r}\|K\|_{\infty}^{r}\left(\int_{\mathcal{B}^{m}}|D h(x-t y)| \mathrm{d} y\right)^{r} \mathrm{~d} t \\
& \leq\|\eta\|_{\infty}^{r}\|K\|_{\infty}^{r} \int_{0}^{\delta} M(D h)(x)^{r} \mathrm{~d} t \\
& \leq c \delta(M D h)(x)^{r} .
\end{aligned}
$$


Estimating II. This is similar, albeit slightly more involved than, the proof of 3(a). First note that

$$
\begin{aligned}
\left(\left|\eta(t) \int_{\mathcal{B}^{m}} t^{-1} h(x-t y) D K(y) \mathrm{d} y\right|\right)^{r} & \leq\left(\|\eta\|_{\infty}\|D K\|_{\infty} t^{-1} \int_{\mathcal{B}^{m}}|h(x-t y)| \mathrm{d} y\right)^{r} \\
& \leq c\left(t^{-1} \cdot t^{-\frac{m}{q}}\|h\|_{L^{q}\left(\mathbb{R}^{m}\right)}\right)^{r} \\
& =c t^{-\frac{(m+q) r}{q}}\|h\|_{L^{q}\left(\mathbb{R}^{m}\right)}^{r} .
\end{aligned}
$$

Here we have used Jensen's Inequality just as in the proof of $3(\mathrm{a})$, but now we have an extra $t^{-1}$ term to incorporate.

Now assume $0<\delta<1$ and consider $\int_{\delta}^{1} t^{-\frac{(m+q) r}{q}} \mathrm{~d} t$ :

$$
\begin{aligned}
\int_{\delta}^{1} t^{-\frac{(m+q) r}{q}} \mathrm{~d} t & =\frac{1}{1-\frac{(m+q) r}{q}}\left[t^{1-\frac{(m+q) r}{q}}\right]_{\delta}^{1} \\
& =\frac{q}{(m+q) r-q}\left[t^{1-\frac{(m+q) r}{q}}\right]_{1}^{\delta} \\
& \leq c \delta^{1-\frac{(m+q) r}{q}} .
\end{aligned}
$$

Now note that $\delta^{1-\frac{(m+q) r}{q}} \leq \delta^{-1}$ if and only if

$$
1-\frac{(m+q) r}{q} \geq-1
$$

i.e.

$$
q \geq \frac{m r}{2-r}
$$

Therefore, from (4.4), and for such $q$, we get

$$
I I \leq c \int_{\delta}^{1} t^{-\frac{(m+q) r}{q}}\|h\|_{L^{q}\left(\mathbb{R}^{m}\right)}^{r} \mathrm{~d} t \leq c \delta^{-1}\|h\|_{L^{q}\left(\mathbb{R}^{m}\right)}^{r} .
$$

Now note that even if $\delta \geq 1$, then $I I \leq 0$, so clearly (4.5) is also true in this case.

Combining these estimates for $I$ and $I I$, we obtain

$$
\int_{0}^{1}\left|D_{x}(\mathbf{E} h)(x, t)\right|^{r} \mathrm{~d} t \leq c \delta(M D h)(x)^{r}+c \delta^{-1}\|h\|_{L^{q}\left(\mathbb{R}^{m}\right)}^{r} .
$$

If we take

$$
\delta=\left(\frac{\|h\|_{L^{q}\left(\mathbb{R}^{m}\right)}^{r}}{(M D h)(x)^{r}}\right)^{\frac{1}{2}}
$$

then (4.6) becomes

$$
\int_{0}^{1}\left|D_{x}(\mathbf{E} h)(x, t)\right|^{r} \mathrm{~d} t \leq c(M D h)(x)^{\frac{r}{2}}\|h\|_{L^{q}\left(\mathbb{R}^{m}\right)}^{\frac{r}{2}} .
$$

Note that whereas the choice of $\delta$ to obtain (4.7) may depend on $x$, the constant $c$ in (4.7) is independent of $x$. So (4.7) holds for all $x \in \mathbb{R}^{m}$. Define the function $\Phi:[0, \infty) \rightarrow[0, \infty)$ as follows:

$$
\Phi(t):= \begin{cases}t^{2} & \text { if } t \in[0,1), \\ t^{\frac{r}{2}} & \text { if } t \geq 1 .\end{cases}
$$


Then $\Psi$ as defined in (4.1) satisfies, for $t \geq 1$,

$$
\Psi(t)=t^{\frac{r}{2}}+t+t \int_{1}^{t} s^{\frac{r}{2}-2} \mathrm{~d} t=t^{\frac{r}{2}}+t+\frac{2}{2-r}\left(1-t^{\frac{r}{2}-1}\right) t \leq c t .
$$

Now apply Lemma 4.2 , noting that $\{|D h| \geq 1\} \subseteq\{\mathbf{M}(D h) \geq 1\}$ :

$$
\begin{aligned}
\int_{\{|D h| \geq 1\}}(M D h)(x)^{\frac{r}{2}} \mathrm{~d} x & \leq \int_{\{M D h \geq 1\}}(M D h)(x)^{\frac{r}{2}} \mathrm{~d} x \\
& =\int_{\{M D h \geq 1\}} \Phi((M D h)(x)) \mathrm{d} x \\
& \leq 2 \times 5^{m} \int_{\{M D h \geq 1\}} \Psi(2|(D h)(x)|) \mathrm{d} x \\
& \leq 2 \times 5^{m} \times 2 c \int_{\{M D h \geq 1\}}|(D h)(x)| \mathrm{d} x \\
& \leq c \int_{\mathbb{R}^{m}}|(D h)(x)| \mathrm{d} x .
\end{aligned}
$$

Therefore, applying (4.8) to (4.7), we obtain

$$
\int_{\{|D h| \geq 1\}} \int_{0}^{1}\left|D_{x}(\mathbf{E} h)(x, t)\right|^{r} \mathrm{~d} t \mathrm{~d} x \leq c\|h\|_{L^{q}\left(\mathbb{R}^{m}\right)}^{\frac{r}{2}} \cdot \int_{\mathbb{R}^{m}}|(D h)(x)| \mathrm{d} x .
$$

As observed in the proof of $3(\mathrm{a})$, if $h$ is compactly supported in $\{x:|x| \leq R\}$, then $\mathbf{E} h$ (and also $D_{x} \mathbf{E} h$ ) is supported in $\{(x, t):|x| \leq R+1, t \in(0,1)\}$. Therefore we have

$$
\int_{\{|D h|<1\}} \int_{0}^{1}\left|D_{x}(\mathbf{E} h)(x, t)\right|^{r} \mathrm{~d} t \mathrm{~d} x \leq\left(\|\eta\|_{\infty}\|K\|_{\infty}\left|\mathcal{B}^{m}\right|\right)^{r} \cdot \mathscr{L}^{m}(\{x:|x| \leq R+1\}) .
$$

Combining (4.9) and (4.10) gives

$$
\int_{\mathbb{R}_{+}^{n}}\left|D_{x}(\mathbf{E} h)(x, t)\right|^{r} \mathrm{~d}(x, t) \leq c+c\|h\|_{L^{q}\left(\mathbb{R}^{m}\right)}^{\frac{r}{2}} \cdot \int_{\mathbb{R}^{m}}|(D h)(x)| \mathrm{d} x .
$$

Since $\mathbf{E}$ is a linear operator, 3(b) easily follows from (4.11) by taking a larger constant $c$.

Acknowledgements. The author is supported by the Oxford Centre for Nonlinear PDE (OxPDE) through the EPSRC Science and Innovation award EP/E035027/1. He would like to thank an anonymous referee for reading a preliminary version of the manuscript and providing thorough feedback, including an extension that has enabled the main result to be stated in a sharper form than it was previously. He is also thankful to Jan Kristensen for numerous helpful discussions.

\section{REFERENCES}

[1] E. Acerbi and G. Dal Maso, New lower semicontinuity results for polyconvex integrals, Calc. Var. Partial Differ. Equ. 2 (1994) 329-371.

[2] L. Ambrosio and G. Dal Maso, On the relaxation in $\operatorname{BV}\left(\Omega ; \mathbf{R}^{m}\right)$ of quasi-convex integrals. J. Funct. Anal. 109 (1992) $76-97$.

[3] L. Ambrosio, N. Fusco, and D. Pallara, Functions of bounded variation and free discontinuity problems, Oxford Mathematical Monographs. The Clarendon Press Oxford University Press, New York (2000).

[4] J.M. Ball and F. Murat, $W^{1, p}$-quasiconvexity and variational problems for multiple integrals. J. Funct. Anal. 58 (1984) $225-253$.

[5] G. Bouchitté, I. Fonseca and J. Malý, The effective bulk energy of the relaxed energy of multiple integrals below the growth exponent. Proc. of R. Soc. Edinburgh Sect. A 128 (1998) 463-479. 
[6] L. Carbone and R. De Arcangelis, Further results on $\Gamma$-convergence and lower semicontinuity of integral functionals depending on vector-valued functions. Ric. Mat. 39 (1990) 99-129.

[7] M. Carozza, J. Kristensen and A. Passarelli di Napoli, Lower semicontinuity in a borderline case. Preprint (2008).

[8] R. Črný, Relaxation of an area-like functional for the function $\frac{x}{|x|}$. Calc. Var. Partial Differ. Equ. 28 (2007) $203-216$.

[9] B. Dacorogna, Direct methods in the calculus of variations. Appl. Math. Sci. 78 (1989).

[10] B. Dacorogna, I. Fonseca, J. Malý and K. Trivisa, Manifold constrained variational problems. Calc. Var. Partial Differ. Equ. 9 (1999) 185-206.

[11] I. Fonseca and J. Malý, Relaxation of multiple integrals below the growth exponent. Ann. Inst. Henri Poincaré, Anal. Non Linéaire 14 (1997) 309-338.

[12] I. Fonseca and J. Malý, From Jacobian to Hessian: distributional form and relaxation. Riv. Mat. Univ. Parma 4 (2005) 45-74.

[13] I. Fonseca and P. Marcellini, Relaxation of multiple integrals in subcritical Sobolev spaces. J. Geom. Anal. 7 (1997) 57-81.

[14] I. Fonseca and S. Müller, Quasi-convex integrands and lower semicontinuity in L1. SIAM J. Math. Anal. 23 (1992) $1081-1098$.

[15] I. Fonseca and S. Müller, Relaxation of quasiconvex functionals in $\operatorname{BV}\left(\Omega, \mathbf{R}^{p}\right)$ for integrands $f(x, u, \nabla u)$. Arch. Ration. Mech. Anal. 123 (1993) 1-49.

[16] I. Fonseca, G. Leoni and S. Müller, A-quasiconvexity: weak-star convergence and the gap. Ann. Inst. Henri Poincaré, Anal. Non Linéaire 21 (2004) 209-236.

[17] L. Greco, T. Iwaniec and G. Moscariello, Limits of the improved integrability of the volume forms. Indiana Univ. Math. J. 44 (1995) 305-339.

[18] T. Iwaniec and G. Martin, Geometric function theory and non-linear analysis. Oxford Mathematical Monographs. The Clarendon Press Oxford University Press, New York (2001).

[19] J. Kristensen, Lower semicontinuity of quasi-convex integrals in BV $\left(\Omega ; \mathbf{R}^{m}\right)$. Calc. Var. Partial Differ. Equ. 7 (1998) $249-261$.

[20] J. Malý, Weak lower semicontinuity of polyconvex integrals. Proc. of R. Soc. Edinburgh Sect. A 123 (1993) 681-691.

[21] J. Malý, Weak lower semicontinuity of polyconvex and quasiconvex integrals. Preprint (1993).

[22] J. Malý, Lower semicontinuity of quasiconvex integrals. Manusc. Math. 85 (1994) 419-428.

[23] P. Marcellini, On the definition and the lower semicontinuity of certain quasiconvex integrals. Ann. Inst. Henri Poincaré, Anal. Non Linéaire 3 (1986) 391-409.

[24] N.G. Meyers, Quasi-convexity and lower semi-continuity of multiple variational integrals of any order. Trans. Amer. Math. Soc. 119 (1965) 125-149.

[25] S. Müller, On quasiconvex functions which are homogeneous of degree 1. Indiana Univ. Math. J. 41 (1992) $295-301$.

[26] F. Rindler, Lower semicontinuity and Young measures in BV without Alberti's rank-one theorem. Adv. Calc. Var. 5 (2012) $127-159$.

[27] W. Rudin, Real and complex analysis, 3rd edition, McGraw-Hill Book Co., New York (1987).

[28] J. Serrin, A new definition of the integral for nonparametric problems in the calculus of variations. Acta Math. 102 (1959) 23-32.

[29] J. Serrin, On the definition and properties of certain variational integrals. Trans. Amer. Math. Soc. 101 (1961) $139-167$.

[30] V. Šverák, Quasiconvex functions with subquadratic growth. Proc. of R. Soc. London A 433 (1991) $723-725$.

[31] K. Zhang, A construction of quasiconvex functions with linear growth at infinity. Ann. Scuola Norm. Sup. Pisa Cl. Sci. (4) 19 (1992) 313-326.

[32] W.P. Ziemer, Weakly differentiable functions, Sobolev spaces and functions of bounded variation. Graduate Texts in Mathematics 120 (1989). 